\section{The incidence of injury in elite (IAAF World Championship) athletes is $97 / 1000$ athletes, with lower limb injuries accounting for $80 \%$ of all injuries; muscle strains are the most type of injury}

Title: Sports injuries surveillance during the 2007 IAAF World Athletics Championships Authors: Alonso JM, Junge A, Renstrom P, Engebretsen L, Mountjoy M, Dvorak J

Reference: Clin J Sport Med 2009; 19(1): 26-32

Type of study: Prospective cohort study

Keywords: injury incidence, track and field, top-level athletes, championships

EB Rating: 8/10, Cl Rating: 7/10

Background: There are reports of a high annual incidence of injuries in athletics, but this has not been studied well

Research question/s: What is the incidence and nature of all sports injuries that were incurred in competitions and/or training during the 2007 World Athletics Championships?

\section{Methodology:}

- Subjects: 1660/1980 (84\%) of registered athletes (various disciplines) participating in the 11th IAAF World Athletics Championships

- Experimental procedure: Previously validated injury report forms $(n=333)$ were completed by team physicians and physiotherapists of 49 teams during the event. 192 injuries (defined as "all musculo-skeletal injuries newly occurred during competition or training regardless of the consequences with respect to absence from competition or training") were reported. All injuries and time-loss injuries were reported,

- Measures of outcome: Incidence of injury (per 1000 athletes), anatomical location, type, injuries in different disciplines,

Main finding/s:

- Incidence of injuries: 97/1000 registered athletes, with 72.4 /1000 during competition

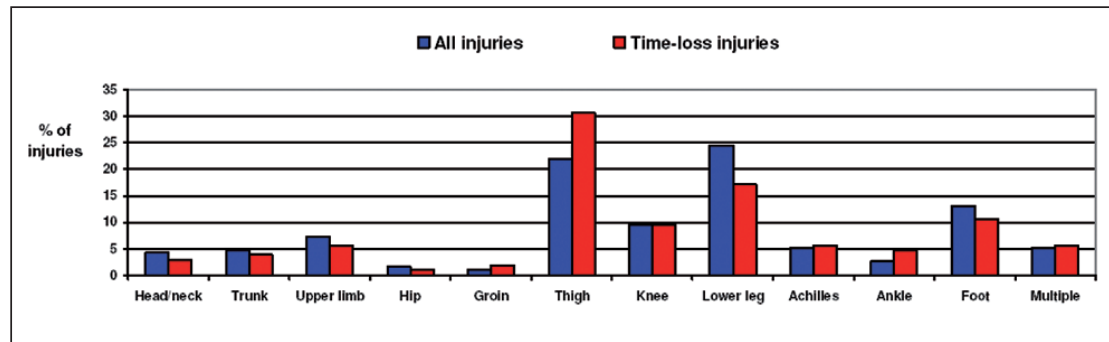

- Most injuries were overuse (44\%) and most occurred in the lower limbs (80\%) - the most common injury was a thigh strain (15\%)

- Injuries by discipline: This varied and the highest risk of a time-loss injury was heptathIon, women's 10,000 m, women's 3000 m steeplechase, decathlon, and men's marathon

Conclusion/s:

- The incidence of injury in elite (IAAF World Championship) athletes is 97/1000 athletes, with lower limb injuries accounting for $80 \%$ of all injuries - muscle strains are the most type of injury

Methodological considerations:

Well conducted study, results may not be applicable to non-elite athletes

SMU Volume 9 (2) 1 p2: 2009 Category: Injury / Athletics / Epidemiology

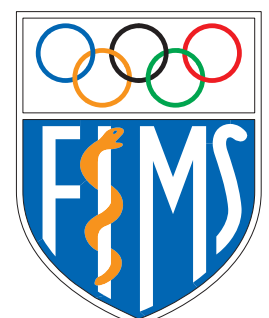

